\title{
Conhecimentos em nutrição dos enfermeiros do curso de especialização em Saúde da Família
}

\author{
Knowledge of the postgraduate health family program nurses on nutrition
}

\author{
Christiane Gonçalves Leite ${ }^{1}$, Grazielle Christine Maciel Mattos ${ }^{2}$, Nínive de Almeida Reis ${ }^{3}$, \\ Ana Cristina Viana Campos ${ }^{4}$
}

\section{RESUMO}

Objetivo: Analisar o perfil e os conhecimentos de nutrição dos enfermeiros alunos do curso de especialização em Saúde da Família em uma universidade de Minas Gerais.

Materiais e Métodos: Estudo transversal descritivo com os enfermeiros $(n=18)$ inseridos no curso de pós-graduação em uma universidade de Minas Gerais, Brasil. A coleta de dados foi realizada por meio da aplicação de questionários. $O$ primeiro foi composto por informações sócio-demográficas. O segundo instrumento continha 20 questões sobre conhecimentos gerais em nutrição selecionadas pelos autores por meio de revisão da literatura específica para o tema abordado. Para o questionário sobre nutrição, foi realizado teste-reteste e teste Alpha de Cronbach por dois profissionais nutricionistas $(\alpha=0,95)$. A construção do banco de dados e análise descritiva foi realizada no programa estatístico SPSS versão 17.

Resultados: As variáveis sociodemográficas que mais contribuíram para a formação dos clusters foram: estado civil $(p=0,040)$ e presença do nutricionista na equipe $(p=0,001)$. O cluster 1 foi formado por enfermeiros do sexo feminino $(76,5 \%)$, solteiras $(66,7 \%)$, que residem no município $(90,9 \%)$, trabalham na estratégia de saúde da família $(69,2 \%)$ e não tem nutricionista na equipe (100,0\%). A associação entre o número de acerto sobre conhecimentos de nutrição e os clusters foi realizada pelo teste Mann-Whitney. A média de acertos foi 10,9 $( \pm 1,5)$ no cluster 1 e 13,8 $( \pm 1,9)$ no cluster 2 , sendo que a diferença foi estatisticamente significante $(p=0,021)$.

Conclusão: Conclui-se que os enfermeiros que contam com nutricionistas na equipe apresentaram conhecimentos adequados relacionados a diferentes aspectos da nutrição.

Palavras-chave: nutrição em saúde pública; programa saúde da família; enfermagem.

\section{ABSTRACT}

Objective: To analyze the profile and the nutritional knowledge of nurse students specializing in Family Health in a University of Minas Gerais.

Materials and Methods: Cross sectional study with nurses $(n=18)$ from the postgraduate program at a university in Minas Gerais, Brazil. Data collection was performed trough questionnaire application. The first one was composed of socio-demographic information. The second instrument contained 20 questions on general knowledge in nutrition, selected by the authors through a specific literature review on the subject. In the nutrition questionnaire analyses, we carried out test-retest reliability and Cronbach's alpha test for two professional nutritionists $(\alpha=0.95)$. The construction of the database and descriptive analysis was performed in SPSS version 17.

Results: The sociodemographic variables that contributed most to the formation of the clusters were: marital status $(p=0.040)$ and the presence of a nutritionist on staff $(p=0.001)$. The first cluster was formed by female nurses $(76.5 \%)$, not married (66.7\%), living in the county (90.9\%), working in the family health strategy (69.2\%) and that had no nutritionist in the team (100.0\%). The association between the number of correct answers about nutrition knowledge and the clusters was performed by the Mann-Whitney test. The mean score was $10.9( \pm 1.5)$ in cluster 1 and $13.8( \pm 1.9)$ in cluster 2, and the difference was statistically significant $(p=0.021)$.

Conclusion: We concluded that nurses who have nutritionists on staff had adequate knowledge related to different aspects of nutrition.

Keywords: nutrition; public health; family health program; nursing.

\footnotetext{
${ }^{1}$ Nutricionista. Especialista em Saúde da Família pelo Instituto de Educação Continuada da Pontifícia Universidade Católica de Minas Gerais (PUCMG).

${ }^{2}$ Cirurgiã-dentista. Doutoranda em Saúde Coletiva pela Faculdade de Odontologia da Universidade Federal de Minas Gerais (UFMG).

${ }^{3}$ Nutricionista formada pela Universidade Federal de Viçosa.

${ }^{4}$ Cirurgiã-dentista. Doutoranda em Saúde Coletiva pela Faculdade de Odontologia da UFMG. Professora do Instituto de Educação Continuada da PUCMG.
} 


\section{INTRODUÇÃO}

A Estratégia Saúde da Família (ESF) foi proposta pelo Ministério da Saúde (MS) como uma ferramenta de reorganização da atenção básica e possibilidade de reorientação do sistema de saúde, incorporando os princípios da universalização, descentralização, integralidade e participação da comunidade. A equipe básica da ESF é composta por um médico, um enfermeiro, um auxiliar de enfermagem e de quatro a seis agentes comunitários de saúde ${ }^{1}$. No formato ampliado, é formada também por um dentista, um auxiliar de consultório dentário e um técnico em higiene dental $^{2}$.

As ações abrangem a assistência integral ao individuo, e prioritariamente, atividades de promoção da saúde e prevenção de doenças para população adstrita3. Dentre estas, a educação em saúde é a de maior destaque pelo seu alcance e pelo custo beneficio favorável.

$\mathrm{Na}$ área de nutrição, trabalhos mais recentes apontam que a educação nutricional não deve mais ser realizada por meio de folhetos ou livretos, nem ser concebidos apenas como a mudança de hábitos alimentares desconsiderando as questões sociais ${ }^{4}$. A educação nutricional é apontada como estratégia no intuito de conter os avanços da ocorrência de doenças crônicodegenerativas, considerando que uma alimentação de má qualidade é considerada um fator de risco para inúmeras doenças ${ }^{5}$.

A ausência do nutricionista na maioria das equipes de saúde no Brasil $^{6}$ pode fazer com que outros profissionais sejam responsabilizados por ações de orientação e aconselhamento aos usuários portadores de comorbidades advindas de hábitos alimentares da população como diabéticos, hipertensos, obesos, desnutridos, de todas as faixas etárias. Quando presente na equipe de saúde, o nutricionista tende a acumular funções em diferentes setores e há predomínio das atividades assistenciais em detrimento de atividades de promoção à saúde ${ }^{7}$.

Nesse contexto, cabe refletir se os enfermeiros estão preparados e tem conhecimentos suficientes para prestar orientações nutricionais à população. Assim, o objetivo do presente estudo foi analisar o perfil e os conhecimentos de nutrição dos enfermeiros alunos do curso de especialização em Saúde da Família em uma universidade de Minas Gerais.

\section{MATERIAIS E MÉTODOS}

Este é um estudo transversal com os enfermeiros inseridos no curso de pós-graduação em Saúde da Família de uma universidade em Minas Gerais, Brasil. Todos os enfermeiros foram convidados e aceitaram participar deste estudo $(n=18)$. Os critérios de inclusão foram os que se seguem: a) estar presente em sala de aula no dia da aplicação do questionário, b) possuir graduação em enfermagem e c) ter assinado o Termo de Consentimento Livre e Esclarecido.

A anuência em participar dessa pesquisa foi voluntária e anônima, sendo que todos os participantes concordaram em assinar um Termo de Consentimento Livre e Esclarecido contendo informações completas referentes aos objetivos e às justificativas da pesquisa, conforme orientações da Resolução $196 / 96^{8}$ que determina as diretrizes das pesquisas envolvendo seres humanos. O projeto de pesquisa foi aprovado pelo Comitê de Ética em Pesquisa da Pontifícia Universidade Católica de Minas Gerais sob o parecer CAAE 0150.0.213.000-10.

A coleta de dados foi realizada no mês de julho de 2010, na própria sala de aula durante o intervalo por meio da aplicação de dois questionários. O primeiro foi composto por informações sócio-demográficas: idade, sexo (masculino, feminino), estado civil (casado, divorciado, solteiro), residência (no município do estudo, outro município mineiro), tipo de vínculo profissional (Saúde da Família e outros) e presença do nutricionista na equipe (sim, não). O segundo instrumento continha 20 questões sobre conhecimentos gerais em nutrição selecionadas pelos autores por meio de revisão da literatura específica para o tema abordado ${ }^{9-14}$. Esse questionário era do tipo autoaplicável com questões estruturadas, escolhidas em função do objetivo, da população de estudo e da viabilidade da coleta dos dados. As variáveis avaliadas foram: questões relacionadas à infância (aleitamento materno, crescimento e desnutrição) e questões gerais (indicação de alimentação, fonte de ferro, problemas nutricionais, e 
risco de obesidade).

Foi realizado teste-reteste para o questionário sobre nutrição, mas não foi realizado o processo de validação do mesmo devido ao tamanho da amostra. A confiabilidade foi testada pelo teste Alpha de Cronbach por dois profissionais nutricionistas $(\alpha=0,95)$. Um grupo de discussão foi organizado para definir por consenso as alternativas corretas para cada questão.

A construção do banco de dados foi realizada no programa estatístico Statistical Package for the Social Sciences for Windows - SPSS versão 17 para análise dos mesmos. Inicialmente, foi realizada a análise descritiva das variáveis sobre os conhecimentos relacionados à nutrição (frequências relativa e absoluta). Apenas as respostas corretas foram descriminadas nas tabelas, sendo que as demais respostas foram agrupadas na categoria "outros". Definido o critério de correção para as questões, o número de acertos foi somado para cada participante, sendo que esses valores poderiam oscilar entre 0 e 20.

Posteriormente, foi realizada a análise de segmentação cujo objetivo principal é definir a estrutura dos dados, alocando os casos mais similares no mesmo grupo, baseado nas características em que os dados foram medidos. O método Two Step Cluster foi utilizado para agrupar a amostra a partir da distância Euclidiana, tendo como critério de agregação das respostas, o critério centróide. Neste tipo de análise, os casos são agrupados por dissimilaridade, ou seja, quanto menores o valores observados, mais parecidos são os casos ${ }^{15}$.

As variáveis sóciodemográficas foram usadas para formar os clusters, sendo que a idade foi escolhida como centroide, por ser uma variável contínua. Nesse tipo de análise, o teste de Bonferroni Ajustado foi usado para medir o peso das variáveis e as diferenças externas entre os clusters formados. A comparação entre os clusters formados e o número de acertos sobre nutrição foi feita por meio do teste Mann-Whitney, com nível de significância estabelecido em $p \leq 0,05$.

\section{RESULTADOS}

As variáveis sociodemográficas que mais contribuíram para a formação dos clusters foram: estado civil $(p=0,040)$ e presença do nutricionista na equipe $(p=0,001)$. O cluster 1 foi formado por enfermeiros do sexo feminino $(76,5 \%)$, solteiras $(66,7 \%)$, que residem no município (90,9\%), trabalham na $\operatorname{ESF}(69,2 \%)$ e não tem nutricionista na equipe $(100,0 \%)$ (tabela 1$)$.

A tabela 2 mostra que nenhum enfermeiro sabia que 0 objetivo da diluição do leite de vaca para lactantes é reduzir teor de sais minerais e proteínas. Outro erro averiguado foi em relação à contra-indicação de reidratação oral em crianças desnutridas, pois apenas $16,7 \%$ marcaram diarreia persistente.

Para a maioria dos enfermeiros, a contraindicação de aleitamento materno é ingurgitamento mamário (88,9\%), crianças com menos de 1 ano de idade não devem consumir mel $(66,7 \%)$, as principais intercorrências capazes de interferir no crescimento de pré-escolares são desmame precoce, diarreia crônica, desnutrição $(77,8 \%)$, déficit de estatura para a idade em crianças indica desnutrição crônica (83,3\%) e o desmame precoce foi considerada a principal causa de desnutrição em bebês com menos de 6 meses (72,2\%) (tabela 2).

Todos os enfermeiros responderam corretamente as questões sobre as comorbidades provindas da obesidade e exemplos de alimentos usados na dietoterapia da anemia. Os principais erros foram nas questões relacionadas em que a minoria marcou aves e suínos como fontes de infecção por salmonela (22,2\%), cárie dentária bócio endêmico como problemas nutricionais no Brasil (33,3\%) e que a carência de proteína e vitamina A podem causar lesões cutâneas em Kwashiorkor (16,7\%) (tabela 3).

A associação entre o número de acerto sobre conhecimentos de nutrição e os clusters foi realizada pelo teste Mann-Whitney. A média de acertos foi 10,9 $( \pm 1,5)$ no cluster 1 e $13,8( \pm 1,9)$ no cluster 2 , sendo que a diferença foi estatisticamente significante $(p=0,021)$ (figura 1).

\section{DISCUSSÃO}

Tradicionalmente, o enfermeiro é o profissional de saúde que aplica conhecimento aliado ao cuidado e à afetividade para melhorar as condições gerais do indivíduo no seu contexto cultural, social, emocional ${ }^{9}$. A maioria dos enfermeiros deste estudo é jovem $(34,3 \pm 5,6$ 
TABELA 1 - Distribuição dos clusters e do total dos participantes em relação às características sócio-demográficas e vínculo profissional $(n=18)$.

\begin{tabular}{|c|c|c|c|c|}
\hline \multirow[t]{2}{*}{ Variáveis } & \multicolumn{2}{|c|}{ Clusters } & \multirow{2}{*}{$\begin{array}{c}\text { Total } \\
(n=18)\end{array}$} & \multirow[t]{2}{*}{$\mathrm{p}(\text { valor })^{\star \star}$} \\
\hline & $1(n=14)$ & $2(n=04)$ & & \\
\hline \multirow[t]{2}{*}{ Idade $^{*}$} & $33,7 \pm 5,3$ & $36,6 \pm 6,8$ & $34,3 \pm 5,6$ & 0,505 \\
\hline & n (\%) & n (\%) & n (\%) & \\
\hline \multicolumn{5}{|l|}{ Sexo } \\
\hline Feminino & $13(76,5)$ & $11(23,5)$ & $17(94,4)$ & 0,467 \\
\hline Masculino & $01(100)$ & $00(0)$ & $01(5,6)$ & \\
\hline \multicolumn{5}{|l|}{ Estado civil } \\
\hline Solteiro & $06(66,7)$ & $03(33,3)$ & $09(50)$ & \\
\hline Casado & $08(100)$ & $00(0)$ & $08(44,4)$ & 0,040 \\
\hline Divorciado & $00(0)$ & $01(100)$ & $01(5,6)$ & \\
\hline \multicolumn{5}{|l|}{ Endereço } \\
\hline Município & $10(90,9)$ & $01(9,1)$ & $11(61,1)$ & 0,137 \\
\hline Outro município MG & $04(57,1)$ & $03(42,9)$ & $07(38,9)$ & \\
\hline \multicolumn{5}{|l|}{ Vínculo profissional } \\
\hline PSF & $09(69,2)$ & $04(100)$ & $13(72,2)$ & 0,234 \\
\hline Outros & $05(100)$ & $00(0)$ & $05(27,8)$ & \\
\hline \multicolumn{5}{|l|}{ Nutricionista na equipe } \\
\hline Sim & $02(33,3)$ & $04(66,7)$ & $06(33,3)$ & 0,001 \\
\hline Não & $12(100)$ & $00(0)$ & $12(66,7)$ & \\
\hline
\end{tabular}

*Valores apresentados em média ( \pm desvio padrão). ${ }^{* *}$ Teste de Bonferroni Ajustado.

anos de idade) e do sexo feminino (94,4\%). Esses resultados são semelhantes a outros ${ }^{16,17}$. Historicamente, se percebe que o processo de construção da identidade profissional de enfermagem é marcado pelas relações de gênero feminino sobre o cotidiano assistencial e organizativo da enfermagem base no sexo feminino ${ }^{17}$.

Para verificar se havia assimetria entre os enfermeiros em relação ao perfil sociodemográfico, foi realizada a análise de segmentação ${ }^{15}$, que dividiu os participantes em dois grupos distintos. O centróide escolhido pela análise foi a idade, a partir da qual as outras diferenças entre os participantes foram surgindo. De acordo coma análise de segmentação, a maioria dos enfermeiros desse estudo é mulheres jovens, casadas que trabalham na Saúde da Família no município do estudo e não tem nutricionista na equipe.

Quando o agrupamento dos dados é bem sucedido, os indivíduos pertencentes ao mesmo grupo são mais semelhantes entre si, tornando os grupos homogêneos internamente e com alta heterogeneidade externa. Apesar de o método Hierárquico ser o mais apropriado para análises com reduzido número de casos, para o presente estudo optou-se pelo método Two Step, uma vez que o mesmo oferece a possibilidade de manipular as variáveis contínuas e categóricas ${ }^{15}$.

Os clusters apresentaram diferenças no perfil sócio demográfico em relação à média de acertos sobre os conhecimentos em nutrição, mesmo que a amostra seja pequena e homogênea. Os resultados do teste MannWhitney mostraram que os enfermeiros deste estudo mais jovens, que já atuem na ESF, mas sem nutricionistas na equipe cometeram mais erros quando questionados sobre conceitos de nutrição $(p=0,021)$.

Poucas unidades de saúde e de Saúde da Família contam com o nutricionista ${ }^{7,18}$ e a maioria da população não procura frequentemente esse profissional. Essa tarefa acaba sendo transferida ao enfermeiro, que pode não ter embasamento técnico-científico específico adequado e também é sobrecarregado de funções. Por isso, há necessidade de um maior esclarecimento dos enfermeiros acerca desses cuidados. Nesta perspectiva, a capacitação profissional em relação ao estado nutricional da população e suas implicações na mesma se fazem extremamente necessária, pois esses profissionais atuam na linha de frente dos serviços 
TABELA 2 - Frequência absoluta e relativa da resposta correta e de respostas inadequadas ("outros") dos enfermeiros para questões relacionadas à infância $(n=18)$.

\begin{tabular}{|c|c|c|c|}
\hline Variáveis & Cluster $1(n=14)$ & Cluster $2(n=04)$ & Total $(n=18)$ \\
\hline & n (\%) & n (\%) & n (\%) \\
\hline \multicolumn{4}{|l|}{ Objetivo da diluição do leite de vaca } \\
\hline Reduzir teor de sais minerais e proteínas & $00(0)$ & $00(0)$ & $00(0)$ \\
\hline Outros & $14(100)$ & $04(100)$ & $18(100)$ \\
\hline \multicolumn{4}{|l|}{ Contraindicação de aleitamento materno } \\
\hline Ingurgitamento mamário & $13(92,9)$ & $03(75)$ & $16(88,9)$ \\
\hline Outros & $01(7,1)$ & $01(25,0)$ & $02(11,1)$ \\
\hline \multicolumn{4}{|l|}{ Causas do desmame precoce e tardio } \\
\hline Nenhuma das respostas & $07(50)$ & $04(100)$ & $11(61,1)$ \\
\hline Outros & $07(50)$ & $00(0)$ & $07(38,9)$ \\
\hline \multicolumn{4}{|l|}{ Contraindicação alimentar para 1 ano de idade } \\
\hline Mel & $09(64,3)$ & $03(75)$ & $12(66,7)$ \\
\hline Outros & $05(35,7)$ & $01(25)$ & $06(33,3)$ \\
\hline \multicolumn{4}{|l|}{ Causas crescimento de pré-escolares } \\
\hline Desmame precoce, diarreia crônica, desnutrição & $11(78,6)$ & $03(75)$ & $14(77,8)$ \\
\hline Outros & $03(21,4)$ & $01(25)$ & $04(22,2)$ \\
\hline \multicolumn{4}{|l|}{ Déficit de estatura para a idade em crianças } \\
\hline Desnutrição crônica & $11(78,6)$ & $04(100)$ & $15(83,3)$ \\
\hline Outros & $03(21,4)$ & $00(0)$ & $03(16,7)$ \\
\hline \multicolumn{4}{|l|}{ Principal causa de desnutrição $\leq 6$ meses } \\
\hline Desmame precoce & $09(64,3)$ & $04(100)$ & $13(72,2)$ \\
\hline Outros & $05(35,7)$ & $00(0)$ & $05(27,8)$ \\
\hline $\begin{array}{l}\text { Contraindicação de reidratação oral em crianças } \\
\text { desnutridas }\end{array}$ & & & \\
\hline Diarreia persistente & $02(14,3)$ & $01(25)$ & $03(16,7)$ \\
\hline Outros & $12(85,7)$ & $03(75)$ & $15(83,3)$ \\
\hline
\end{tabular}

públicos de saúde.

Esse problema também acontece em outras áreas. Os médicos e enfermeiros em São Paulo admitiram pouco saber sobre nutrição e que a maior causa era a falta de disciplinas sobre nutrição na graduação ${ }^{19}$. Isso acontece, provavelmente porque o ensino de nutrição na graduação não contempla as demandas da prática profissional $^{9,20}$.

É preciso considerar a possibilidade de uma formação mais humana que privilegie uma visão integral do homem, voltada para um ensino interdisciplinar ${ }^{5}$. Diferentemente da área da saúde, a educação vem se preocupando em discutir e implantar propostas metodológicas para abordar melhor as questões da alimentação saudável de crianças em todas as idades ${ }^{21-}$ 24. Experiências de capacitação de professores e educação em saúde para escolares realizada por nutricionistas têm mostrado grandes avanços como aumento do conhecimento sobre alimentação e nutrição ${ }^{25}$, diminuição de consumo de lipídios, de proteínas e de colesterol $^{26}$, estímulo ao desenvolvimento de habilidades culinárias e instrumentalização para as escolhas e práticas alimentares $^{27}$.

O cluster 2 formado por apenas quatro enfermeiros que contam com nutricionistas na equipe acertaram mais questões sobre nutrição, média de 13,8 ( $\pm 1,9)$. Entretanto, não é possível realizar inferências sobre este grupo, devido ao numero reduzido de casos. Os enfermeiros deste estudo demonstraram ter algumas informações sobre a relação alimento e indivíduo, mas em muitos aspectos, há falta de informação e de autonomia sobre o assunto. Esses resultados apontam a necessidade de reforçar o trabalho interdisciplinar buscando mudanças no lidar com as questões rotineiras próprias da vida da população e que, muitas vezes, 
TABELA 3 - Frequência absoluta e relativa da resposta correta e de respostas inadequadas ("outros") dos enfermeiros para questões gerais $(n=18)$.

\begin{tabular}{|c|c|c|c|}
\hline Variáveis & Cluster $1(n=14)$ & Cluster 2 ( $n=04)$ & Total $(n=18)$ \\
\hline & n (\%) & n (\%) & n (\%) \\
\hline \multicolumn{4}{|l|}{ Fonte de ferro } \\
\hline Feijões/espinafre/mariscos/fígado & $13(92,9)$ & $02(50)$ & $15(83,3)$ \\
\hline Outros & $01(7,1)$ & $02(50)$ & $03(16,7)$ \\
\hline \multicolumn{4}{|l|}{ Fontes de infecção por salmonella } \\
\hline Aves e suínos & $02(14,3)$ & $02(50)$ & $04(22,2)$ \\
\hline Outros & $12(85,7)$ & $02(50)$ & $14(77,8)$ \\
\hline \multicolumn{4}{|l|}{ Problemas nutricionais no Brasil } \\
\hline Outros & $10(71,4)$ & $02(50)$ & $12(66,7)$ \\
\hline Cárie Dental e Bócio Endêmico & $04(28,6)$ & $02(50)$ & $06(33,3)$ \\
\hline \multicolumn{4}{|l|}{ Aumento de ferro em gestantes } \\
\hline Maior síntese de hemoglobina & $06(42,9)$ & $03(75)$ & $09(50)$ \\
\hline Outros & $06(57,1)$ & $01(25)$ & $09(50)$ \\
\hline \multicolumn{4}{|l|}{ Maior risco de anemia } \\
\hline Gestantes e crianças $<6$ anos & $09(64,3)$ & $04(100)$ & $13(72,2)$ \\
\hline Outros & $05(35,7)$ & $00(0)$ & $05(27,8)$ \\
\hline \multicolumn{4}{|l|}{ Causa de lesões cutâneas em Kwashiorkor } \\
\hline Outros & $11(78,6)$ & $04(100)$ & $15(83,3)$ \\
\hline Proteína e vitamina A & $03(21,4)$ & $00(0)$ & $03(16,7)$ \\
\hline \multicolumn{4}{|l|}{ Pós-operatório de fenda palatina } \\
\hline Evitar uso de mamadeira & $10(71,4)$ & $03(75)$ & $13(72,2)$ \\
\hline Outros & $04(28,6)$ & $01(25)$ & $05(27,8)$ \\
\hline \multicolumn{4}{|l|}{ Alteração por chá e café } \\
\hline Ação estimulante & $13(92,9)$ & $04(100)$ & $17(94,4)$ \\
\hline Outros & $01(7,1)$ & $00(0)$ & $01(5,6)$ \\
\hline \multicolumn{4}{|l|}{ Indicação de alimentação dietoterápica } \\
\hline Anemia & $14(100)$ & $04(100)$ & $18(100)$ \\
\hline \multicolumn{4}{|l|}{ Fator de risco de obesidade } \\
\hline Hipertensão arterial e diabetes mellitus não insulino-dependente & $14(100)$ & $04(100)$ & $18(100)$ \\
\hline
\end{tabular}

pode passar despercebida aos olhos de um único profissional de saúde. Para isso, é necessário que cada categoria saia do estatuto científico estreito para uma nova conformação de equipe, considerando o processo atual do trabalho em saúde ${ }^{9}$. Por outro lado, a formação acadêmica do enfermeiro e sua sobrecarga de trabalho torna difícil sua dedicação às atividades voltadas para a alimentação, prevenção e promoção da saúde ligada à educação nutricional da população.

Alguns estudos tem discutido a importância da nutrição no ensino de graduação na Medicina e na Enfermagem no Brasil ${ }^{19,28,29}$, bem como a atuação e importância do nutricionista na ESF ${ }^{30,31}$. Entretanto, este parece ser um dos poucos estudos que investigou a percepção e entendimento de enfermeiros pósgraduandos em relação aos conhecimentos em nutrição por meio de um questionário elaborado com boa confiabilidade.

Esse estudo apresenta algumas limitações relevantes. O desenho metodológico transversal do presente estudo não permite que conclusões de causa efeito sejam assumidas. Outra limitação diz respeito à utilização de uma amostra de conveniência, mesmo com 100\% de taxa de resposta. Além disso, com uma divisão dos clusters 1 e 2 em 14 e 04 enfermeiros, respectivamente, não foi possível discutir as diferenças nos tipos de respostas para cada questão. Por último, não é possível comparar o nível de conhecimento em nutrição dos 


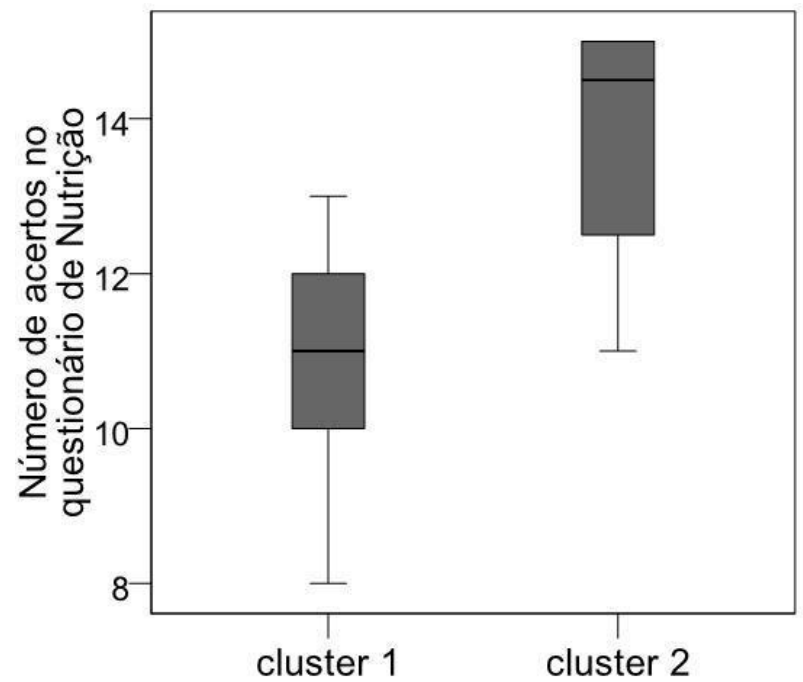

FIGURA 1 - Medidas de resumo e tendência central para o teste de Mann-Whitney entre o cluster $1(n=14)$ e cluster 2 $(n=04)$ em relação ao número de acertos sobre conhecimentos de nutrição $(p=0,021)$.

enfermeiros ou qualquer outro profissional de saúde com os nutricionistas.

Conclui-se que os enfermeiros que contam com nutricionistas na equipe apresentaram conhecimentos adequados relacionados a diferentes aspectos da nutrição. Os resultados obtidos neste estudo apontam para uma abordagem multidisciplinar e discussões sobre a formação e o perfil profissional que desejamos para Saúde da Família. Portanto, cabe aos nutricionistas atuarem como facilitadores, promovendo cursos e programas de educação continuada nos serviços de saúde em que atuam de tal forma que o conhecimento seja disseminado e possa abranger os enfermeiros $e$ outros profissionais da saúde. Espera-se que esse trabalho seja um caminho para outros estudos que atentam para este tema impreterivelmente necessário no cenário da saúde brasileira.

\section{REFERÊNCIAS}

1. Brasil. Ministério da Saúde. Saúde da família: uma estratégia para a reorientação do modelo assistencial. Brasília (DF): Ministério da Saúde, 1997. [capturado 2010 Mar 20] Disponível em: http://bvsms.saude.gov.br/ bvs/publicacoes/cd09_16.pdf

2. Brasil. Ministério da Saúde. Cadernos de Atenção Básica; Obesidade. Brasília (DF): Ministério da Saúde; 2006. [capturado 2010 Mar 20] Disponível em: http://bvsms.saude.gov.br/bvs/publicacoes/abcad12.pdf

3. Brasil. Ministério da Saúde. Secretaria de Atenção à Saúde. Departamento de Atenção Básica. Política nacional de atenção básica. Brasília (DF): Ministério da Saúde; 2006. [capturado 2010 Mar 20] Disponível em: http://bvsms.saude.gov.br/bvs/publicacoes/politica_nacion al_atencao_basica_2006.pdf

4. Manço AM, Costa FNA. Educação nutricional: caminhos possíveis. Alim Nutr. 2004;15(2):145-53.

5. Amorim STSP, Moreira H, Carraro TE. A formação de pediatras e nutricionistas: a dimensão humana. Rev Nutr. 2001; 14(2):111-8.

6. Boog MCF. Atuação do nutricionista em saúde pública na promoção da alimentação saudável. Rev Ciência \& Saúde 2008; 1(1):33-42.

7. Pádua JG, Boog MCF. Avaliação da inserção do nutricionista na Rede Básica de Saúde dos municípios da Região Metropolitana de Campinas. Rev Nutr. 2006; 19(4):413-24.

8. Brasil. Ministério da Saúde. Resolução n.196, de 10 de outubro de 1996. Diretrizes e normas regulamentadoras de pesquisas envolvendo seres humanos. Brasília (DF): Ministério da Saúde; 1996.

9. Campos SH, Boog MCF. Cuidado nutricional na visão de enfermeiras docentes. Rev Nutr. 2006; 19(2):145-55.

10. Escott-Stump S, Mahan, L. K. Krause. Alimentos, nutrição e dietoterapia. $11^{\circ}$ ed. São Paulo: Roca; 2005.

11. Franco AC, Boog MCF. Relação teoria-prática no ensino de educação nutricional. Rev Nutr. 2007; 20(6):643-55.

12. World Health Organization. Global strategy on diet, physical activity and health. Fifty-Seventh World Health Assemblies - WHA57.17. Geneva: WHO; 2004. [capturado 2011 Jul 27]. Disponível em: http://www.who.int/ dietphysicalactivity/strategy/eb11344/strategy_english_we b.pdf

13. Brasil. Ministério da Saúde. Política nacional de alimentação e nutrição. $2^{\text {a }}$ ed. Brasília (DF): Ministério da Saúde; 2003. [capturado 2010 Mar 20] Disponível em: http://bvsms.saude.gov.br/bvs/publicacoes/pnan.pdf

14. Guimarães FPM, Takayanagui AMM. Orientações recebidas do serviço de saúde por pacientes para o tratamento do portador de Diabetes Mellitus tipo 2. Rev Nutr. 2002; 15(1):37-44.

15. Hair JF, Black WC, Babin JB, Anderson RE, Tatham RL. Análise multivariada de dados. $6^{\mathrm{a}}$ ed. Porto Alegre: Artmed; 2009.

16. Santos LSC, Guirardello EB. Demandas de atenção do enfermeiro no ambiente de trabalho. Rev Latino-Am. Enfermagem 2007; 15(1)27-33.

17. Moreira MCN. Imagens no espelho de vênus: mulher, enfermagem e modernidade. Rev Latino-Am. Enfermagem 1999; 7(1):55-65.

18. Assis AMO, Santos SMC, Freitas MCS, Silva MCM. O programa saúde da família: contribuições para uma reflexão sobre a inserção do nutricionista na equipe multidisciplinar. Rev Nutr. 2002; 15(3):255-66.

19. Boog MCF, Roncada MJ, Stewien G. Ensino de nutrição nos cursos de Medicina e Enfermagem no Estado de São Paulo. Acta Paul Enferm. 1995; 8(4):66-75.

20. Boog MCF. Educação nutricional: passado, presente, futuro. Rev Nutr. 1997;10(1):5-19

21. Boog MCF. Programa de educação nutricional em escola de ensino fundamental de zona rural. Rev Nutr. 2010; 23(6):1005-17.

22. Bernardon R, Silva JRM, Cardoso GT, Monteiro RA, Amorim NFA, Schmitz BAS et al. Construção de metodologia de capacitação em alimentação e nutrição para educadores. Rev Nutr. 2009; 22(3):389-98.

23. Bizzo MLG, Leder L. Educação nutricional nos parâmetros curriculares nacionais para o ensino fundamental. Rev Nutr. 2005;18(5):661-7.

24. Teixeira TC, Sigulem DM, Correia IC. Avaliação dos 
conteúdos relacionados à nutrição contidos nos livros didáticos de biologia do ensino médio. Rev Paul Pediatr. 2011; 29(4):560-6.

25. Detregiachi CRP, Braga TMS. Projeto "criança saudável, educação dez": resultados com e sem intervenção do nutricionista. Rev Nutr. 2011; 24(1):51-9.

26. Cervato AM, Derntl AM, Latorre MRDO, Marucci MFN. Educação nutricional para adultos e idosos: uma experiência positiva em Universidade Aberta para a Terceira Idade. Rev Nutr. 2005; 18(1):41-52.

27. Castro IRR, Souza TSN, Maldonado LA, Caniné ES, Rotenberg S, Gugelmin SA. A culinária na promoção da alimentação saudável: delineamento e experimentação de método educativo dirigido a adolescentes e a profissionais das redes de saúde e de educação. Rev Nutr. 2007; 20(6):571-88.

28. Feresin C, Sonzogno MC. Reflexões sobre a inserção da disciplina de nutrição na formação do enfermeiro. Rev Latino-Am Enfermagem [periódico online]. 2007 nov-dez [capturado 2011 Jul 27]; 15(6). Disponível em: http://www.eerp.usp.br/rlae.
29. Boog MCF. Construção de uma proposta de ensino de nutrição para curso de enfermagem. Rev Nutr. 2002; 15(1):15-28.

30. Geus LMM, Maciel CS, Burda ICA, Daros SJ, Batistel S, Martins TCA et al. A importância na inserção do nutricionista na Estratégia Saúde da Família. Ciênc Saúde Colet 2011; 16(Supl. 1):797-804.

31. Santos AC. A inserção do nutricionista na Estratégia da Saúde da Família: o olhar de diferentes trabalhadores da saúde. Fam Saúde Desenv 2005; 7(3):257-65.

\section{Endereço para correspondência}

Ana Cristina Viana Campos

Rua dos Jês 151, apto. 302

Belo Horizonte/MG - CEP 31530-160

Telefone: +553197715409

E-mail: campos.acv@gmail.com 